\title{
Aggressiveness of Isolates of Phytophthora infestans from the Columbia Basin of Washington and Oregon
}

\author{
Jeffrey S. Miller, Dennis A. Johnson, and Philip B. Hamm
}

First and second authors: Department of Plant Pathology, Washington State University, Pullman 99164-6430; and third author: Hermiston Agricultural Research and Extension Center, Department of Botany and Plant Pathology, Oregon State University, Hermiston 97838. Accepted for publication 29 November 1997.

\begin{abstract}
Miller, J. S., Johnson, D. A., and Hamm, P. B. 1998. Aggressiveness of isolates of Phytophthora infestans from the Columbia Basin of Washington and Oregon. Phytopathology 88:190-197.

The aggressiveness of 22 isolates of Phytophthora infestans collected from naturally infected potato plants in the Columbia Basin of Washington and Oregon was determined on detached potato leaflets at $18^{\circ} \mathrm{C}$ in an incubator. Selected isolates were evaluated on whole plants in a greenhouse. Aggressiveness was measured by using the area under the lesion expansion curve (AULEC), incubation period, latent period, sporulation capacity, and lesion size on detached leaflets and the area under the disease progress curve and sporulation capacity on whole plants. The detached-leaflet assay was useful in that a large number of isolates were tested, several components of aggressiveness were studied, and signifi-

cant differences were found among isolates. Significant variation for components of aggressiveness was found within and among isolates classified according to genotype. Significant interactions among isolates and cultivars were found for some components of aggressiveness, so results were pooled according to cultivar. On average, US- 8 and US-11 isolates had higher AULEC scores, indicating aggressiveness higher than that of US-7, US-6, and US-1 genotypes. One US-8 genotype isolate had a higher standardized sporulation capacity than isolates of the other genotypes. US-6 genotype isolates were the least aggressive group, as indicated by low AULEC, sporulation capacity, and lesion size values. The replacement of the US- 1 genotype by the US- 8 genotype in the Columbia Basin may be partially explained by the increased aggressiveness of US-8 isolates. Additionally, potato growers may need to shorten intervals between fungicide applications and begin applications earlier.
\end{abstract}

Late blight, caused by Phytophthora infestans (Mont.) de Bary, is one of the most destructive diseases of the cultivated potato (Solanum tuberosum subsp. tuberosum L.). In the Columbia Basin of Washington and Oregon, late blight was first documented in 1947 by J. D. Menzies (1). From 1947 to 1990, the population of $P$. infestans in this area was believed to be composed of a single clonal lineage $(9,10)$ characterized by the A1 compatibility type and sensitivity to the systemic fungicide metalaxyl. The situation was found to have changed when new strains insensitive to metalaxyl were detected in 1991 (7) and when the A2 compatibility type was detected in 1993 (15). Metalaxyl-insensitive isolates outnumbered metalaxyl-sensitive isolates 31 to 1 in 1993, even though metalaxyl-sensitive isolates were dominant in 1992 (15). Similar replacement of the original A1 metalaxyl-sensitive population by newer A2 compatibility type strains has recently occurred in the Netherlands, Germany, and Poland $(9,33)$. A recent study examining the appearance of new strains in the United States found 11 different multilocus genotypes from 1992 to 1993 (12). Nine of these genotypes had never been detected in the United States or Canada prior to that study. Only four of the genotypes had been detected from more than three samples (12). Many factors, including increased aggressiveness and metalaxyl insensitivity, may have contributed to the replacement of the old clonal lineage by newer strains.

Many studies have documented differential aggressiveness among isolates of $P$. infestans. Castronovo et al. (3) reported differential aggressiveness in terms of infectivity, incubation period, and sporulation in 1954. Later studies showed that differences in infectivity among isolates of the same race on whole plants were

Corresponding author: J. S. Miller; E-mail address: jsmiller@mail.wsu.edu

Publication no. P-1997-1229-02R

(c) 1998 The American Phytopathological Society just as great as differences among isolates of different races (24). Other investigators, using intact plants, detached leaflets, and tuber disks, showed that isolates typically differ for characters related to aggressiveness (20-23,35) and considered these fitness components. Differences in relative aggressiveness have been observed between metalaxyl-insensitive and metalaxyl-sensitive isolates on agar, intact plants, and detached leaves $(2,6,20-22)$ and between isolates from the old clonal lineage and A1 compatibility type isolates from the sexually reproducing population found in Mexico on intact plants (35). Another study, in which detached leaflets were used, compared isolates from US-1, US-7, and US-8 genotypes and showed that US-7 and US-8 isolates had shorter latent periods than US-1 isolates and that US-8 isolates produced larger lesions and more sporangia than US-1 isolates (23). Lambert and Currier recently reported that US-6, US-7, and US-8 isolates caused tubers to rot substantially faster than US-1 isolates (25).

Laboratory testing of $P$. infestans on potato plant parts has made it possible to test several isolates simultaneously $(8,16)$. In particular, detached-leaflet assays have been useful in investigations with $P$. infestans and potato to identify $\mathrm{R}$ genes $(8,32,34)$, screen for pathogenicity (13), quantify partial resistance in potato cultivars and clones (30), identify aggressive isolates of $P$. infestans $(23,30)$, and study various ratios and combinations of A1 and A2 compatibility types in oospore production (5). Levels of infection and response to spore concentration have also been tested on detached leaflets $(27,32)$. Levels of host resistance determined by detached-leaflet assays were similar to levels of observed field resistance $(32,34)$. These studies indicate that the use of detached leaflets can save time and space and allow for testing more $P$. infestans isolates. Additionally, the results of these tests correlate well with field results $(32,34)$.

The magnitude of variation for various components of aggressiveness among isolates of $P$. infestans in the Columbia Basin is not known. This study was conducted to quantify aggressiveness of isolates collected from the Columbia Basin to test the hypothe- 
sis that differential aggressiveness has played a significant role in the replacement of the old clonal lineage by new strains. Information gained in this study could be used to improve the timing of fungicide applications.

\section{MATERIALS AND METHODS}

Potato plants. Certified seed tubers were used to establish potato plants for each experiment. The cultivars Russet Burbank, Russet Norkotah, Shepody, and Legend (formerly COO8-3008-1) were chosen to test isolates against a range of cultivars used in the Columbia Basin. These cultivars vary in their levels of partial resistance; Legend is the most resistant (17) and Russet Norkotah the least. Seed tubers were rinsed under warm tap water, surface sterilized in $1 \%$ sodium hypochlorite for $10 \mathrm{~min}$, and then allowed to dry under ambient laboratory conditions. Tubers were left on the laboratory bench for 5 to 7 days to promote sprout formation. Sprouting buds were cut from tubers with a sterile, dish-shaped, melon-ball cutter to produce spherical tuber seed pieces weighing approximately $13 \mathrm{~g}$ each. Tuber seed pieces were planted at a depth of $10 \mathrm{~cm}$ in $15-\mathrm{cm}$-diameter plastic pots containing a standard potting mix (3:1:1 [vol/vol/vol], peat moss, pumice, and sand). Plants were watered upon emergence, and fertilizer was applied weekly with an irrigation solution containing $500 \mathrm{ppm}$ of NPK, 20-20-20. A 16-h photoperiod was maintained by using sodium lamps with an approximate intensity of 200 to $225 \mu \mathrm{mol} \mathrm{m} \mathrm{m}^{-2} \mathrm{~s}^{-1}$.

Isolate collection, maintenance, and characterization. Isolates of $P$. infestans were obtained from naturally infected potato foliage in the Columbia Basin of Washington and Oregon from 1992 to 1994, except isolate 537, which was collected from potatoes in western Washington, and isolate 444B, which was collected from tomato in the Columbia Basin. Cultures were obtained by transferring sporangia from the margin of a single sporulating lesion onto rye extract agar (29) amended with $100 \mu \mathrm{g}$ each of penicillin, pimaricin, and polymyxin per milliliter or by removing small areas of infected leaf tissue near the lesion margin and plating it onto rye extract agar containing $10 \mu \mathrm{l}$ of pimaricin per milliliter, $250 \mathrm{U}$ of ampicillin per milliliter, and $10 \mu \mathrm{g}$ of rifampicin per milliliter. Isolates were derived by transferring a hyphal tip or single sporangium to unamended rye extract agar. Isolates were maintained on rye extract agar at $18^{\circ} \mathrm{C}$ in darkness and were transferred every 4 to 5 weeks. Fresh cultures ( 1 to 3 weeks old) were used to infect potato tuber slices to increase sporangia for inoculum. All isolates from 1992 and 1993 were inoculated on potato tuber slices or plant foliage and then reisolated at least once to maintain pathogenicity before increasing inoculum on Russet Norkotah tuber slices.

Each isolate was tested for compatibility type, metalaxyl sensitivity, and glucose 6-phosphate isomerase (Gpi) allozyme genotype. Compatibility types were determined by pairing each isolate against two known A1 and two known A2 testers on rye extract agar. Agar plates were kept in the dark at $18^{\circ} \mathrm{C}$ for 14 days and then examined for oospores with a dissecting microscope. Metalaxyl sensitivity of isolates was evaluated by placing isolates singly on plates containing rye extract agar amended with $0.1,1.0,10.0$, and $100.0 \mu \mathrm{g}$ of technical grade metalaxyl per milliliter. Classification of strains as sensitive or resistant was determined following a modification of the scheme used by Deahl et al. by using $10.0 \mu \mathrm{g}$ of metalaxyl per milliliter as the critical value (8). Sensitive and resistant isolates displayed $\leq 10$ and $>10 \%$ growth, respectively, compared with the control. Isolates Fi 2 (US-1) and 382 (US-1) were used as standards for sensitive responses and 584 (US-8) and 537 (US-11) as standards for insensitive responses. These standards were chosen because of their vigorous growth on rye extract agar in the absence of metalaxyl.

Isolates were analyzed for Gpi allozyme genotype (11). Mycelia were scraped from 2- to 3-week-old cultures with a spatula and placed into $1.5-\mathrm{ml}$ centrifuge tubes containing $1 \mathrm{ml}$ of deionized distilled water. Tubes were stored on ice between steps for the remainder of the procedure. After centrifugation at $13,000 \times g$ for $5 \mathrm{~min}$, approximately half the supernatant in each tube was discarded. The pellet in each tube was ground with a plastic pestle attached to a high-speed, electric, hand-held drill until the sample mixture was homogenized. Tubes were again centrifuged at $13,000 \times g$ for $5 \mathrm{~min}$. Aliquots of supernatant were then electrophoresed and stained with agar overlays for Gpi (11). The following standards were used: Fi 2 (US-1), Pi 94 (US-6), 301 and 302 (US-7), 584 (US-8), and 537 (US-11) (28). Only two or three standards were run per plate, depending on the phenotype of the isolates being compared, i.e., 584, 301, and 302 with A2 isolates; 537 and Pi 94 with A1 metalaxyl-resistant isolates; and $\mathrm{Fi} 2$ with $\mathrm{A} 1$ metalaxyl-sensitive isolates. When a combination of phenotypes was analyzed, a combination of appropriate standards was used.

Inoculum production. Russet Norkotah potato tubers were surface sterilized as in the seed-piece preparation and, after drying, were sliced transversely into disks $1 \mathrm{~cm}$ thick. Tuber slices were placed in $20-\times 100-\mathrm{mm}$ glass Petri dishes containing a $9-\mathrm{cm}$ Whatman no. 1 filter paper moistened with $5 \mathrm{ml}$ of deionized distilled water. A 50- $\mu$ l drop of unquantified sporangial solution obtained from a 1- to 3-week-old agar culture was placed on the section immediately after slicing. Cultures on tuber slices were then incubated at $18^{\circ} \mathrm{C}$ in darkness for 6 to 7 days. Since some isolates sporulated sooner than others, a second set of tuber disks was inoculated the day after preparation of the first set, so that sporangia of similar maturity could be used for inoculation.

Detached-leaflet studies. The area under the lesion expansion curve (AULEC), incubation period, latent period, sporulation capacity, and lesion size were used to evaluate isolate aggressiveness on detached leaflets. Leaflets from the fourth to sixth subterminal leaves of test plants were detached from greenhousegrown plants and were selected so that all leaflets were of similar age and size. Leaflets were placed abaxial side up on moistened filter paper in glass Petri dishes $(15 \times 100 \mathrm{~mm})$, and each was inoculated with a $25-\mu \mathrm{l}$ drop of sporangial suspension adjusted to a concentration of $1 \times 10^{4}$ sporangia per milliliter. A single leaflet was used as an experimental unit, and four to five replications were conducted per test.

Leaflets were then placed in an incubator at $18^{\circ} \mathrm{C}$ with a $16-\mathrm{h}$ photoperiod (57 to $59 \mu \mathrm{mol} \mathrm{m} \mathrm{m}^{-2} \mathrm{~s}^{-1}$ ) and examined $24 \mathrm{~h}$ after inoculation, then every $12 \mathrm{~h}$ until 4 days postinoculation, and then every $24 \mathrm{~h}$ until 7 days postinoculation. At each observation, severity was rated by assessing the proportion of the leaflet with visible disease symptoms. Ratings were made according to a 0 to 4 scale developed by Cohen (4), where 0 indicated no symptoms and 4 represented 96 to $100 \%$ of the leaflet surface blighted. Incubation period and latent period, the time from inoculation until initial necrosis, and the time from inoculation until sporangia first appeared (31) were noted. AULEC was computed from data collected daily. At 7 days postinoculation, lesion areas were measured by placing overhead transparencies over the Petri dishes and tracing around the edges of the lesions. The lesion area tracings on the overhead transparencies were measured by using an image analysis system (Decagon Devices, Agvision, Pullman, WA). Sporangia were then collected by placing leaflets in 50-ml polystyrene bottles with approximately $10 \mathrm{ml}$ of sporangial fixative (90:5:5 [vol/vol/vol], ethanol, glacial acetic acid, and formalin) and shaking the bottles for $5 \mathrm{~min}$ on an Orbit Shaker (Lab-Line Instruments, Melrose Park, IL) at 1,500 rpm to dislodge sporangia. The concentration of sporangia in fixative was determined with a hemacytometer (eight counts per leaflet). The total number of sporangia produced per lesion was calculated and then divided by the lesion area to obtain the sporulation capacity. Sporulation capacity and lesion size were not measured in all tests.

Whole-plant studies. Five isolates representing four clonal lineages (US-1, US-7, US-8, and US-11) were further tested for aggressiveness on whole potted plants of Russet Norkotah and 
Legend in the greenhouse. Area under the disease progress curve (AUDPC) and sporulation capacity were used as measures of aggressiveness. Plants 6 to 8 weeks old were uniformly inoculated with a sporangial suspension of $P$. infestans at a concentration of $1 \times 10^{4}$ sporangia per milliliter with a hand-held mini-spray gun (Sears, Roebuck and Co., Chicago, IL). Each plant had three stems with five to six fully expanded leaves per stem. A volume of $50 \mathrm{ml}$ of sporangial solution was evenly applied over five plants. Plants were then immediately placed into mist chambers. Care was taken to prevent plants inoculated with different isolates from touching.
Incubation in mist chambers was at ambient temperatures of 15 to $20^{\circ} \mathrm{C}$ for $20 \mathrm{~h}$. After incubation, plants were moved to greenhouse benches and evaluated every $12 \mathrm{~h}$ from $36 \mathrm{~h}$ to 5 days postinoculation and every $24 \mathrm{~h}$ for 6 and 7 days postinoculation. Five infected leaves were tagged per plant. The percent infection of each leaf was rated on a 0 to 10 scale $(0=$ no infection, $1=10 \%$, $2=20 \%$, etc.), and incubation period was recorded. Daily disease ratings were used to determine AUDPC.

The time periods required for sporulation and isolate sporulation capacity were determined after the final disease rating because relative humidity was not favorable for sporulation in the

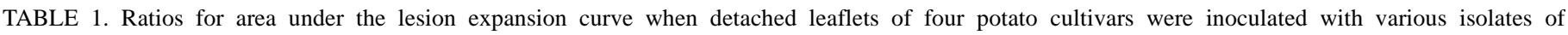
Phytophthora infestans ${ }^{\mathrm{x}}$

\begin{tabular}{|c|c|c|c|c|c|c|c|c|c|}
\hline \multirow[b]{2}{*}{ Isolate } & \multirow[b]{2}{*}{ Genotype } & \multicolumn{2}{|c|}{ Russet Burbank } & \multicolumn{2}{|c|}{ Russet Norkotah } & \multicolumn{2}{|c|}{ Shepody } & \multicolumn{2}{|c|}{ Legend } \\
\hline & & Test 1 & Test 2 & Test 1 & Test 2 & Test 1 & Test 2 & Test 1 & Test 2 \\
\hline 584 & 8 & $3.23 \mathrm{a}$ & $2.03 \mathrm{a}$ & $2.34 \mathrm{ab}$ & $2.19 \mathrm{abc}$ & $1.96 \mathrm{a}$ & $1.52 \mathrm{bc}$ & $1.64 \mathrm{a}$ & $1.68 \mathrm{ab}$ \\
\hline 510 & 8 & $3.10 \mathrm{a}$ & $2.00 \mathrm{a}$ & $2.31 \mathrm{abc}$ & $1.94 \mathrm{abcd}$ & $1.73 \mathrm{ab}$ & $2.09 \mathrm{a}$ & $\ldots$ & $\ldots$ \\
\hline 332 & 11 & $2.47 \mathrm{ab}$ & $\ldots$ & $1.60 \mathrm{abcd}$ & $\ldots$ & $1.73 \mathrm{ab}$ & $\ldots$ & $\ldots$ & $\ldots$ \\
\hline 444B & 7 & $2.26 \mathrm{abc}$ & $1.02 \mathrm{de}$ & $2.15 \mathrm{abc}$ & $2.24 \mathrm{ab}$ & 1.24 bcde & $0.87 \mathrm{de}$ & $\ldots$ & $\ldots$ \\
\hline 537 & 11 & $1.82 \mathrm{bcd}$ & $2.04 \mathrm{a}$ & 1.51 abcde & $2.54 \mathrm{a}$ & $1.53 \mathrm{abcd}$ & $1.21 \mathrm{c}$ & $1.57 \mathrm{a}$ & $1.49 \mathrm{abc}$ \\
\hline 417 & 8 & $1.61 \mathrm{bcd}$ & $1.79 \mathrm{ab}$ & $\ldots$ & $2.56 \mathrm{a}$ & $1.38 \mathrm{bcde}$ & $1.46 \mathrm{bc}$ & $\ldots$ & $\ldots$ \\
\hline $410^{y}$ & $\ldots$ & $1.97 \mathrm{bcd}$ & $1.60 \mathrm{abc}$ & $2.42 \mathrm{a}$ & $1.63 \mathrm{bcde}$ & $1.60 \mathrm{abc}$ & $1.71 \mathrm{ab}$ & $\ldots$ & $\ldots$ \\
\hline 422 & 6 & $1.56 \mathrm{bcd}$ & $1.41 \mathrm{abcd}$ & $1.79 \mathrm{abcd}$ & $2.11 \mathrm{abc}$ & $1.07 \mathrm{de}$ & $0.85 \mathrm{e}$ & $\ldots$ & $\ldots$ \\
\hline 382 & 1 & $1.64 \mathrm{bcd}$ & 1.03 cde & $1.15 \mathrm{defg}$ & $0.95 \mathrm{fg}$ & $0.12 \mathrm{f}$ & $0.11 \mathrm{~g}$ & $0.56 \mathrm{bc}$ & $\ldots$ \\
\hline $345^{y}$ & $\ldots$ & $1.37 \mathrm{cde}$ & $1.52 \mathrm{abcd}$ & $0.76 \mathrm{gh}$ & $0.90 \mathrm{fg}$ & $1.01 \mathrm{de}$ & $1.67 \mathrm{ab}$ & $\ldots$ & $\ldots$ \\
\hline 302 & 7 & $1.27 \mathrm{de}$ & 1.18 cde & 1.33 cdefg & $1.21 \mathrm{efg}$ & 1.17 cde & $1.18 \mathrm{~cd}$ & $1.08 \mathrm{~b}$ & $1.20 \mathrm{bcd}$ \\
\hline 416 & 1 & $1.16 \mathrm{de}$ & $1.07 \mathrm{cde}$ & $1.61 \mathrm{abcd}$ & $1.28 \mathrm{defg}$ & $1.20 \mathrm{bcde}$ & $1.42 \mathrm{bc}$ & $1.73 \mathrm{a}$ & $1.22 \mathrm{bcd}$ \\
\hline 367 & 7 & $1.20 \mathrm{de}$ & 0.79 ef & $1.18 \mathrm{defg}$ & $1.45 \mathrm{cdef}$ & $0.98 \mathrm{e}$ & $1.15 \mathrm{cde}$ & $1.54 \mathrm{a}$ & $1.79 \mathrm{a}$ \\
\hline $336^{y}$ & $\ldots$ & 0.87 ef & 1.24 bcde & $1.98 \mathrm{abc}$ & $1.43 \mathrm{cdef}$ & 1.12 cde & $1.64 \mathrm{ab}$ & $\ldots$ & $\ldots$ \\
\hline 462 & 6 & $\ldots$ & 1.14 cde & & 1.50 bcdef & 1.33 bcde & $1.75 \mathrm{ab}$ & & \\
\hline $\mathrm{Fi} 2$ & 1 & $\ldots$ & $\ldots$ & $0.87 \mathrm{efgh}$ & 1.69 abcde & $\ldots$ & $\ldots$ & $0.81 \mathrm{bc}$ & $1.15 \mathrm{~cd}$ \\
\hline 436 & 6 & $\ldots$ & $1.06 \mathrm{cde}$ & $\ldots$ & $\ldots$ & $1.06 \mathrm{de}$ & $1.32 \mathrm{bc}$ & $\ldots$ & $\ldots$ \\
\hline Fi 1 & 1 & $0.72 \mathrm{ef}$ & $0.59 \mathrm{fg}$ & 1.49 bcdef & $\ldots$ & $\ldots$ & $\ldots$ & $1.56 \mathrm{a}$ & $\ldots$ \\
\hline Pi 63 & 1 & $\ldots$ & $\ldots$ & $0.87 \mathrm{fgh}$ & $\ldots$ & $\ldots$ & $\ldots$ & $0.82 \mathrm{bc}$ & $\ldots$ \\
\hline $466^{z}$ & $\ldots$ & $\ldots$ & $0.43 \mathrm{gh}$ & $\ldots$ & $0.79 \mathrm{~g}$ & $\ldots$ & $0.87 \mathrm{de}$ & $\ldots$ & $0.836 \mathrm{~d}$ \\
\hline 469 & 1 & $0.49 \mathrm{f}$ & $0.23 \mathrm{~h}$ & $0.46 \mathrm{~h}$ & $0.27 \mathrm{~h}$ & $0.33 \mathrm{f}$ & $0.29 \mathrm{f}$ & $0.61 \mathrm{c}$ & $0.24 \mathrm{e}$ \\
\hline
\end{tabular}

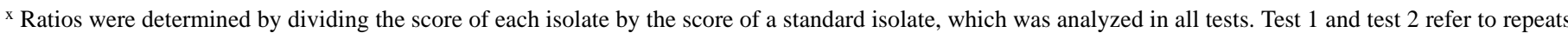
of the same test. Missing data are designated by ellipses. Within a data column, values followed the same letter are not significantly different $(P=0.05)$ according to Duncan's multiple range test. Values are means of four to five replications.

y Isolate does not belong to a currently defined genotype (A1, Gpi 100/111).

${ }^{\mathrm{z}}$ Isolate does not belong to a currently defined genotype (A2, Gpi 100/100).

TABLE 2. Ratios for latent period when detached leaflets of four potato cultivars were inoculated with various isolates of Phytophthora infestans

\begin{tabular}{|c|c|c|c|c|c|c|c|c|c|}
\hline \multirow[b]{2}{*}{ Isolate } & \multirow[b]{2}{*}{ Genotype } & \multicolumn{2}{|c|}{ Russet Burbank } & \multicolumn{2}{|c|}{ Russet Norkotah } & \multicolumn{2}{|c|}{ Shepody } & \multicolumn{2}{|c|}{ Legend } \\
\hline & & Test 1 & Test 2 & Test 1 & Test 2 & Test 1 & Test 2 & Test 1 & Test 2 \\
\hline 584 & 8 & $0.58 \mathrm{a}$ & $0.68 \mathrm{a}$ & 0.78 bcdef & $0.73 \mathrm{abc}$ & $0.75 \mathrm{bc}$ & $0.78 \mathrm{bcd}$ & $0.72 \mathrm{ab}$ & 0.72 \\
\hline 510 & 8 & $0.67 \mathrm{ab}$ & $0.78 \mathrm{a}$ & $0.58 \mathrm{a}$ & $0.74 \mathrm{abc}$ & $0.70 \mathrm{ab}$ & $0.71 \mathrm{bc}$ & $\ldots$ & $\ldots$ \\
\hline 332 & 11 & $0.61 \mathrm{a}$ & $\ldots$ & 0.85 cdefg & $\ldots$ & $0.74 \mathrm{bc}$ & $\ldots$ & $\ldots$ & $\ldots$ \\
\hline 444B & 7 & $0.63 \mathrm{a}$ & $0.80 \mathrm{abc}$ & $0.71 \mathrm{abc}$ & $0.63 \mathrm{a}$ & $0.93 \mathrm{def}$ & $1.08 \mathrm{fg}$ & $\ldots$ & $\ldots$ \\
\hline 537 & 11 & $0.74 \mathrm{abc}$ & $0.69 \mathrm{a}$ & 0.78 bcde & $0.63 \mathrm{a}$ & $0.73 \mathrm{bc}$ & 0.92 efg & $0.73 \mathrm{abc}$ & 0.77 \\
\hline 417 & 8 & 0.93 cde & $0.71 \mathrm{a}$ & $\ldots$ & $0.63 \mathrm{a}$ & 0.98 def & $0.95 \mathrm{efg}$ & $\ldots$ & $\ldots$ \\
\hline $410^{y}$ & $\ldots$ & $0.62 \mathrm{ab}$ & $0.76 \mathrm{ab}$ & $0.65 \mathrm{ab}$ & $0.69 \mathrm{ab}$ & $0.63 \mathrm{a}$ & $0.78 \mathrm{bcd}$ & $\ldots$ & $\ldots$ \\
\hline 422 & 6 & $0.67 \mathrm{ab}$ & $0.69 \mathrm{a}$ & $0.70 \mathrm{abc}$ & $0.63 \mathrm{a}$ & 0.86 cdef & $1.08 \mathrm{fg}$ & $\ldots$ & $\ldots$ \\
\hline 382 & 1 & 0.89 cde & $1.02 \mathrm{~d}$ & $1.08 \mathrm{fgh}$ & $1.07 \mathrm{efg}$ & $1.90 \mathrm{~g}$ & $2.11 \mathrm{~h}$ & $1.17 \mathrm{e}$ & $\ldots$ \\
\hline $345^{y}$ & $\ldots$ & $1.05 \mathrm{de}$ & $0.68 \mathrm{a}$ & $1.09 \mathrm{gh}$ & $0.96 \mathrm{def}$ & $0.97 \mathrm{def}$ & $0.68 \mathrm{~b}$ & $\ldots$ & $\ldots$ \\
\hline 302 & 7 & $0.80 \mathrm{bcd}$ & $0.91 \mathrm{bcd}$ & $0.74 \mathrm{abcd}$ & 0.86 bcde & 0.83 bcde & $0.91 \mathrm{def}$ & 0.88 bcde & 0.79 \\
\hline 416 & 1 & $1.01 \mathrm{de}$ & $0.98 \mathrm{~d}$ & $0.73 \mathrm{abcd}$ & $0.87 \mathrm{bcde}$ & $0.79 \mathrm{bcd}$ & 0.80 cde & $0.64 \mathrm{a}$ & 0.90 \\
\hline 367 & 7 & $1.08 \mathrm{de}$ & $1.04 \mathrm{~d}$ & 0.90 cdefg & $0.76 \mathrm{abcd}$ & $1.00 \mathrm{ef}$ & 0.82 cde & $0.69 \mathrm{ab}$ & 0.72 \\
\hline $336^{y}$ & $\ldots$ & $1.31 \mathrm{ef}$ & $0.75 \mathrm{ab}$ & 0.75 abcd & $0.63 \mathrm{a}$ & $0.97 \mathrm{def}$ & $0.57 \mathrm{a}$ & $\ldots$ & $\ldots$ \\
\hline 462 & 6 & $\ldots$ & $0.94 \mathrm{~cd}$ & $\ldots$ & 0.91 cde & $1.04 \mathrm{f}$ & $0.71 \mathrm{bc}$ & $\ldots$ & $\ldots$ \\
\hline 436 & 6 & $\ldots$ & $0.97 \mathrm{~cd}$ & $\ldots$ & $\ldots$ & $0.70 \mathrm{ab}$ & $0.69 \mathrm{bc}$ & $\ldots$ & $\ldots$ \\
\hline Fi 1 & 1 & $1.28 \mathrm{ef}$ & $1.18 \mathrm{~d}$ & 0.85 cdefg & $\ldots$ & $\ldots$ & $\ldots$ & $0.75 \mathrm{abcd}$ & $\ldots$ \\
\hline Pi 63 & 1 & $\ldots$ & $\ldots$ & 0.96 defg & $\ldots$ & $\ldots$ & $\ldots$ & $1.10 \mathrm{cde}$ & $\ldots$ \\
\hline Fi 2 & 1 & $\ldots$ & $\ldots$ & $1.02 \mathrm{efgh}$ & $0.73 \mathrm{abc}$ & $\ldots$ & $\ldots$ & $1.09 \mathrm{de}$ & 0.99 \\
\hline $466^{z}$ & $\ldots$ & $\ldots$ & $1.74 \mathrm{e}$ & $\ldots$ & $1.31 \mathrm{fg}$ & $\ldots$ & $1.12 \mathrm{~g}$ & $\ldots$ & 1.04 \\
\hline 469 & 6 & $1.70 \mathrm{f}$ & $1.78 \mathrm{e}$ & $1.48 \mathrm{~h}$ & $1.53 \mathrm{~g}$ & $1.85 \mathrm{~g}$ & $1.72 \mathrm{~h}$ & $1.26 \mathrm{e}$ & 1.46 \\
\hline
\end{tabular}

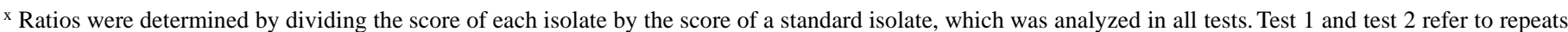
of the same test. Missing data are designated by ellipses. Within a data column, values followed by the same letter are not significantly different $(P=0.05)$ according to Duncan's multiple range test. Values are means of four to five replications.

y Isolate does not belong to a currently defined genotype (A1, Gpi 100/111).

${ }^{\mathrm{z}}$ Isolate does not belong to a currently defined genotype (A2, Gpi 100/100). 
greenhouse. An 8-mm cork borer was used to cut five leaf disks per plant (one disk per leaf). Disks were taken from the leading edge of blight lesions and placed in a $15-\times 100-\mathrm{mm}$ glass Petri dish containing filter paper moistened with deionized distilled water. Leaf disks were incubated at $18^{\circ} \mathrm{C}$ in the light for $3 \mathrm{~h}$ and then examined hourly for sporulation until all leaf disks had sporulated. The time required for sporulation to occur was noted. After $24 \mathrm{~h}$ of incubation, disks were placed in 50-ml polystyrene bottles containing approximately $10 \mathrm{ml}$ of fixative solution and shaken for $5 \mathrm{~min}$ at 1,500 rpm. Sporangia were counted with a hemacytometer as described previously to determine the sporulation capacity.

Experimental design. Detached leaflet tests were designed as randomized complete blocks with four to five replications. Replications were leaflets from different leaves, often from different plants. Whole-plant experiments were arranged in a completely random design with five to six replications. A total of 22 isolates were tested on detached leaflets in nine separate trials with a mean of six isolates per trial. Half of the isolates were included in more than one trial, and all trials were repeated. Since all isolates could not be compared simultaneously, one isolate, Pi 94 (US-6), was chosen arbitrarily as a standard and included in all trials to make relative comparisons among isolates in different trials. Scores of the various aggressiveness components for all isolates tested were standardized by dividing each score by the corresponding mean score of the standard isolate, Pi 94. The combination of the first set of nine trials was labeled test 1 , and the repeated set of trials was labeled test 2 .
A total of five isolates in two trials were tested on whole plants of cultivars Russet Norkotah and Legend grown in the greenhouse. Isolates 537 (US-11), 367 (US-7), and 416 (US-1) were used in the first trial, and isolates 537 (US-11), 584 (US-8), 367 (US-7), and Fi 2 (US-1) were used in the second.

Data were analyzed with the SAS GLM and CORR procedures (SAS Institute, Cary, NC). The residuals of the data set met the assumption of normality with the $\ln (x+1)$ transformation for AULEC, incubation period, sporulation capacity, and lesion size. The reciprocal $(1 / x)$ transformation was used for latent period data. Duncan's multiple range test $(P \leq 0.05)$ was used for comparisons among isolates and among isolates grouped according to genotype. Tests for correlations among variables in detached leaflets were performed by using Pearson's correlation coefficients, and a Spearman's rank correlation was used to compare the performance of isolates on detached leaflets and on whole plants.

\section{RESULTS}

Significant interactions were present among isolates and cultivars. Therefore, isolate aggressiveness scores were grouped according to the cultivar on which they were tested. AULEC, latent period, sporulation capacity, lesion size in detached-leaflet experiments, and AUDPC in whole-plant experiments varied significantly $(P \leq 0.05)$ among isolates (Tables 1-5). Significant differences among isolates for incubation period were found rarely (data not shown). For each component of aggressiveness, isolates

TABLE 3. Ratios for sporulation capacity when detached leaflets of four potato cultivars were inoculated with various isolates of Phytophthora infestans ${ }^{\mathrm{x}}$

\begin{tabular}{|c|c|c|c|c|c|c|c|c|c|}
\hline \multirow[b]{2}{*}{ Isolate } & \multirow[b]{2}{*}{ Genotype } & \multicolumn{2}{|c|}{ Russet Burbank } & \multicolumn{2}{|c|}{ Russet Norkotah } & \multicolumn{2}{|c|}{ Shepody } & \multicolumn{2}{|c|}{ Legend } \\
\hline & & Test 1 & Test 2 & Test 1 & Test 2 & Test 1 & Test 2 & Test 1 & Test 2 \\
\hline 584 & 8 & $\ldots$ & $\ldots$ & 3.76 & 2.85 & $\ldots$ & $\ldots$ & $20.25 \mathrm{a}$ & $5.16 \mathrm{ab}$ \\
\hline Pi 63 & 1 & $\ldots$ & $\ldots$ & 2.64 & $\ldots$ & $\ldots$ & $\ldots$ & $20.06 \mathrm{a}$ & $\ldots$ \\
\hline Fi 2 & 1 & $\ldots$ & $\ldots$ & 3.22 & 2.60 & $\ldots$ & $\ldots$ & $3.87 \mathrm{c}$ & $2.71 \mathrm{~b}$ \\
\hline 537 & 11 & $2.02 \mathrm{bc}$ & $\ldots$ & 1.39 & 1.64 & 1.72 & $\ldots$ & $3.46 \mathrm{c}$ & $2.25 \mathrm{~b}$ \\
\hline $345^{y}$ & $\ldots$ & $2.33 \mathrm{ab}$ & $3.04 \mathrm{a}$ & 1.62 & 1.19 & 1.42 & 1.98 & $\ldots$ & $\ldots$ \\
\hline 302 & 7 & $2.68 \mathrm{a}$ & $2.03 \mathrm{ab}$ & 1.61 & 1.36 & 1.66 & 2.57 & $4.61 \mathrm{c}$ & $7.00 \mathrm{a}$ \\
\hline 416 & 1 & $2.20 \mathrm{bc}$ & $1.53 \mathrm{bc}$ & 1.85 & 1.64 & 1.70 & 1.38 & $10.07 \mathrm{bc}$ & $1.45 \mathrm{ab}$ \\
\hline 367 & 7 & $1.63 \mathrm{bc}$ & $0.81 \mathrm{~cd}$ & 1.92 & 2.14 & 2.03 & 2.18 & $12.57 \mathrm{ab}$ & $2.51 \mathrm{~b}$ \\
\hline $336^{y}$ & $\ldots$ & $1.24 \mathrm{bc}$ & $2.71 \mathrm{ab}$ & 2.23 & 1.30 & 1.91 & 1.63 & $\ldots$ & $\ldots$ \\
\hline Fi 1 & 1 & $1.24 \mathrm{bc}$ & $0.62 \mathrm{~d}$ & 1.77 & $\ldots$ & $\ldots$ & $\ldots$ & $\ldots$ & $\ldots$ \\
\hline $466^{z}$ & $\ldots$ & $\ldots$ & $0.71 \mathrm{~d}$ & $\ldots$ & 1.27 & $\ldots$ & 1.06 & $\ldots$ & $3.99 \mathrm{ab}$ \\
\hline 469 & 1 & $0.84 \mathrm{~b}$ & $0.34 \mathrm{~d}$ & 0.96 & 1.54 & 0.47 & 0.38 & $\ldots$ & $1.80 \mathrm{~b}$ \\
\hline
\end{tabular}

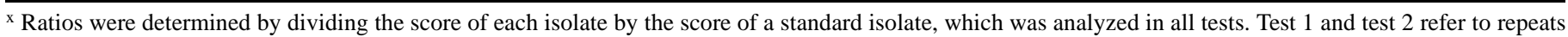
of the same test. Missing data are designated by ellipses. Within a data column, values followed by the same letter are not significantly different $(P=0.05)$ according to Duncan's multiple range test. Values are means of four to five replications.

y Isolate does not belong to a currently defined genotype (A1, Gpi 100/111).

z Isolate does not belong to a currently defined genotype (A2, Gpi 100/100).

TABLE 4. Ratios for lesion size when detached leaflets of four potato cultivars were inoculated with various isolates of Phytophthora infestans ${ }^{\mathrm{x}}$

\begin{tabular}{|c|c|c|c|c|c|c|c|c|c|}
\hline \multirow[b]{2}{*}{ Isolate } & \multirow[b]{2}{*}{ Genotype } & \multicolumn{2}{|c|}{ Russet Burbank } & \multicolumn{2}{|c|}{ Russet Norkotah } & \multicolumn{2}{|c|}{ Shepody } & \multicolumn{2}{|c|}{ Legend } \\
\hline & & Test 1 & Test 2 & Test 1 & Test 2 & Test 1 & Test 2 & Test 1 & Test 2 \\
\hline 584 & 8 & $\ldots$ & $\ldots$ & $2.32 \mathrm{a}$ & 2.27 & $\ldots$ & $\ldots$ & $1.91 \mathrm{a}$ & $2.36 \mathrm{a}$ \\
\hline 537 & 11 & 1.31 & $\ldots$ & $1.42 \mathrm{bc}$ & 1.93 & 1.02 & $\ldots$ & $1.56 \mathrm{abc}$ & $1.73 \mathrm{ab}$ \\
\hline 367 & 7 & 1.40 & 1.66 & $1.27 \mathrm{bc}$ & 1.36 & 1.08 & $1.38 \mathrm{a}$ & $1.52 \mathrm{abc}$ & $2.39 \mathrm{a}$ \\
\hline $345^{y}$ & $\ldots$ & 1.35 & 1.87 & $0.98 \mathrm{~cd}$ & 1.06 & 1.10 & $1.46 \mathrm{a}$ & $\ldots$ & $\ldots$ \\
\hline $336^{y}$ & $\ldots$ & 1.24 & 1.71 & $1.90 \mathrm{ab}$ & 1.71 & 1.23 & $1.63 \mathrm{a}$ & $\ldots$ & $\ldots$ \\
\hline 416 & 1 & 1.17 & 1.33 & $1.68 \mathrm{ab}$ & 1.38 & 1.23 & $1.47 \mathrm{a}$ & $1.66 \mathrm{c}$ & $1.51 \mathrm{~b}$ \\
\hline 302 & 7 & 1.10 & 1.19 & $1.34 \mathrm{bc}$ & 1.27 & 1.04 & $1.10 \mathrm{~b}$ & $\ldots$ & $1.32 \mathrm{~b}$ \\
\hline Fi 1 & 1 & 0.96 & 0.97 & $1.73 \mathrm{ab}$ & $\ldots$ & $\ldots$ & $\ldots$ & $\ldots$ & $\ldots$ \\
\hline Pi 63 & 1 & $\ldots$ & $\ldots$ & $1.54 \mathrm{bc}$ & $\ldots$ & $\ldots$ & $\ldots$ & $1.18 \mathrm{bc}$ & $\ldots$ \\
\hline Fi 2 & 1 & $\ldots$ & $\ldots$ & $0.95 \mathrm{~cd}$ & 1.59 & $\ldots$ & $\ldots$ & $0.17 \mathrm{~d}$ & $0.79 \mathrm{c}$ \\
\hline $466^{z}$ & $\ldots$ & $\ldots$ & 0.68 & $\ldots$ & 0.70 & $\ldots$ & $0.82 \mathrm{c}$ & $\ldots$ & $1.19 \mathrm{bc}$ \\
\hline 469 & 6 & 0.52 & 0.08 & $0.63 \mathrm{~d}$ & 0.46 & 0.22 & $0.13 \mathrm{~d}$ & $\ldots$ & $0.22 \mathrm{~d}$ \\
\hline
\end{tabular}

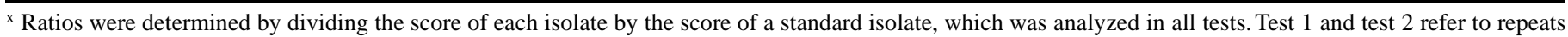
of the same test. Missing data are designated by ellipses. Within a data column, values followed by the same letter are not significantly different $(P=0.05)$ according to Duncan's multiple range test. Values are means of four to five replications.

y Isolate does not belong to a currently defined genotype (A1, Gpi 100/111).

${ }^{\mathrm{z}}$ Isolate does not belong to a currently defined genotype (A2, Gpi 100/100). 
are listed in Tables 1 through 4 from highest AULEC value to lowest according to the response on Russet Burbank in test 1. If two isolates or cultivars did not differ significantly for AULEC values but did exhibit significant differences for test 2 or on another cultivar, then the second score was used for ranking. For whole-plant tests, the same criterion was applied for AUDPC values (Table 5). Some isolates did not infect test leaflets or leaflets decomposed before readings were complete and were scored as missing data.

Two of the US-8 isolates, 584 and 510, had significantly higher mean AULEC values $(P \leq 0.05)$ on the four cultivars used in the tests (Table 1). The third US-8 isolate, 417 , had a high AULEC value on Russet Norkotah leaflets and moderate values on Russet Burbank, Shepody, and Legend. Isolate 537, a US-11 isolate, had relatively high AULEC values on Russet Norkotah, Russet Burbank, and Legend but had moderate AULEC values on Shepody. The AULEC values of the three US-7 isolates were generally lower than those of US- 8 or US-11 isolates. Isolates 302 and 367 had moderate to low AULEC values on all cultivars. Isolate 367 was the only US-7 isolate tested on Legend and had a high AULEC score. Isolate 444B, the isolate from tomato, had a high AULEC value on Russet Norkotah, varied between tests on Russet Burbank, and had a low AULEC score on Shepody. US-1 and US-6 isolates had relatively moderate to low AULEC scores on all cultivars tested.

Four isolates included in the aggressiveness tests, 410, 345, 336 , and 466, possibly arose from sexual recombination (R. W. Sandrock and W. E. Fry, unpublished). Isolate 410 had relatively high AULEC scores on the three cultivars (Table 1). Isolate 345 scored relatively high on Russet Burbank and relatively low on Russet Norkotah and was moderate on Shepody. Isolate 336 varied significantly between tests on all three cultivars and isolate 466 had very low AULEC scores on all cultivars.

The latent period values of isolates followed a trend similar to that of AULEC scores (Table 2), and nontransformed values were significantly correlated (Table 6). Isolates with higher AULEC scores typically had lower latent period values.

Sporulation capacity was highest for isolate 584 on Legend, the only US-8 isolate for which this variable was tested (Table 3). Isolates 302 and 345 had high sporulation capacities on Russet Burbank leaflets. Values for sporulation capacity of isolates on Russet Norkotah and Shepody leaflets were not significantly different. Sporulation capacity was significantly correlated with all other measures of aggressiveness (Table 6).

Lesion size was not as useful in detecting differences among isolates as other variables. Results on Russet Burbank, one test on Russet Norkotah, and one test on Shepody were not significant (Table 4). In tests where results were significant $(P \leq 0.05)$, isolates Fi 2 (US-1), 466 (possible recombinant), and 469 (US-6)

TABLE 5. Aggressiveness components from two tests when various isolates of Phytophthora infestans were inoculated on two potato cultivars in the greenhouse $^{\mathrm{x}}$

\begin{tabular}{|c|c|c|c|c|}
\hline & \multirow[b]{3}{*}{ Genotype } & \multicolumn{2}{|c|}{ Test 1} & \multirow[b]{2}{*}{ Test 2} \\
\hline & & & Sporulation & \\
\hline & & AUDPC $^{y}$ & capacity ${ }^{z}$ & AUDPC \\
\hline \multicolumn{5}{|l|}{ Isolate } \\
\hline 537 & 11 & $62 \mathrm{a}$ & $1.27 \mathrm{a}$ & $118 \mathrm{a}$ \\
\hline 584 & 8 & & & $97 \mathrm{ab}$ \\
\hline 367 & 7 & $35 \mathrm{~b}$ & $0.73 \mathrm{~b}$ & $66 \mathrm{bc}$ \\
\hline Fi 2 & 1 & & & $48 \mathrm{c}$ \\
\hline 416 & 1 & $30 \mathrm{~b}$ & $0.31 \mathrm{~b}$ & $47 \mathrm{c}$ \\
\hline \multicolumn{5}{|l|}{ Cultivar } \\
\hline Legend & & $25 \mathrm{a}$ & $0.29 \mathrm{a}$ & $39 a$ \\
\hline Russet Norkotah & & $71 \mathrm{~b}$ & $0.53 \mathrm{~b}$ & $109 \mathrm{~b}$ \\
\hline
\end{tabular}

${ }^{\mathrm{x}}$ Means within a column and section followed by different letters differ significantly $(P \leq 0.05)$.

y Area under the disease progress curve.

${ }^{\mathrm{z}}$ Number of sporangia $(\times 10,000)$ per square centimeter of lesion area. had small lesion sizes while isolate 584 (US-8) had the largest lesion size. Lesion sizes were significantly correlated to all other variables measured (Table 6).

As a group, US-8 isolates had significantly higher AULEC values $(P \leq 0.05)$ and shorter latent periods on Russet Burbank than US-1, US-6, and US-7 isolates (Table 7). The US-11 isolates were similar to US-8 isolates for AULEC and latent period in most tests and had higher AULEC values and shorter latent periods than US-1 and US-6 isolates in a majority of the tests. No significant differences were found for sporulation capacity on Russet Norkotah and Shepody and for lesion size on Russet Burbank (data not shown). Differences among genotypes were not as great for these two components as for AULEC and latent period.

The isolates representing potential recombinants were classified as having either A1 compatibility type with Gpi 100/111 (isolates 345,410 , and 336) or A2 compatibility type with Gpi 100/100 (isolate 466). The former group had moderate to high AULEC scores, short latent periods, and high sporulation capacity values. The only A2, Gpi 100/100 isolate (isolate 466) scored relatively low for all aggressiveness components.

Because of interactions among isolates and cultivars, the main effect of cultivars could not be analyzed. In general, however, isolates on detached leaflets of Shepody had larger AULEC values, shorter latent periods, and larger sporulation capacity values than isolates tested on the other three cultivars. Isolates on detached leaflets of Russet Burbank and Russet Norkotah had similar aggressiveness values. Isolates tested on Legend had lower AULEC values and longer latent periods than when tested on other cultivars.

Isolates differed significantly for AUDPC and sporulation capacity when tested on whole plants in the greenhouse (Table 5). No interactions were present between isolates and cultivars, so responses were pooled for Legend and Norkotah. In test 1, isolate 537 (US-11) had a significantly higher AUDPC and sporulation capacity than isolates 367 (US-7) and 416 (US-1). When the test was repeated and isolates 584 (US-8) and Fi 2 (US-1) were added, 537 and 584 had higher AUDPC values than the US-1 isolates Fi 2 and 416. Isolate 367 was moderately aggressive.

The AUDPC values for isolates from greenhouse test 2 were compared with the AULEC values for the same five isolates on detached leaflets by using Spearman's rank correlation coefficient. Only AULEC values from Legend and Russet Norkotah detached leaflets were used, since these were the cultivars used in the greenhouse tests. These five isolates ranked similarly $(0.900, P=0.0374)$ on both detached leaflets and whole plants. When a mean AULEC value was determined for isolates grouped by genotype and then compared with the representative isolate in greenhouse test 2, the Spearman's rank correlation coefficient $(0.872, P=0.05)$ was significant.

In whole-plant tests, isolates on Legend had significantly lower AUDPC values $(P \leq 0.0001)$, significantly longer latent periods $(P \leq 0.005)$, and fewer sporangia per lesion area $(P \leq 0.005)$ than isolates on Russet Norkotah (Table 5).

\section{DISCUSSION}

Significant variation for various components of aggressiveness was found among isolates of $P$. infestans collected in the Colum-

TABLE 6. Pearson's correlation coefficients for the relationships among the area under the lesion expansion curve (AULEC), latent period, sporulation capacity, and lesion size when detached potato leaflets were infected with various isolates of Phytophthora infestans ${ }^{\mathrm{z}}$

\begin{tabular}{lccr}
\hline & $\begin{array}{c}\text { Latent } \\
\text { period }\end{array}$ & $\begin{array}{c}\text { Sporulation } \\
\text { capacity }\end{array}$ & $\begin{array}{c}\text { Lesion } \\
\text { size }\end{array}$ \\
\hline AULEC & $-0.71689^{*}$ & $0.28873^{*}$ & $0.79928^{*}$ \\
Latent period & $\ldots$ & $-0.30480^{*}$ & $-0.68954^{*}$ \\
Sporulation capacity & $\ldots$ & $\ldots$ & $0.24785^{*}$ \\
\hline $\mathrm{z} *$ significant at $P \leq 0.0001$. Sample sizes varied from 340 to 698.
\end{tabular}


bia Basin from 1992 to 1994. Significant variation occurred within and among isolates classified according to multilocus genotype. High AULEC, sporulation capacity, and lesion size values and low latent period values represent high aggressiveness. For AULEC, US-8 and US-11 isolates on detached leaflets were typically more aggressive than isolates of other genotypes. The single US-8 isolate measured for sporulation capacity produced more sporangia per unit leaf area than US-1 and US-6 isolates. This observation supports data from previous studies (23). The US-8 isolates were also observed to rot tuber slices faster than other isolates when inoculum was increased, which agrees with the high aggressiveness of US- 8 on potato tubers reported by Lambert and Currier (25). US-11 isolates were just as aggressive as US-8 isolates for AULEC and lesion size. Significant differences were rarely found among US-1, US-7, and US-6 isolates for lesion expansion.

Isolates representing the US-6 genotype had the lowest scores for aggressiveness components on detached leaflets. Isolate 469 consistently was the least aggressive isolate (Tables 1-4). No US-6 isolate ever scored higher than any US-11 or US-8 isolate, and only one of the four US- 6 isolates in the tests was rated as more aggressive than any of the US-7 isolates.

Several of the components chosen to evaluate aggressiveness in this study were reliable in detecting significant differences among isolates of $P$. infestans on detached leaflets. AULEC, representing host tissue colonization over time, required several readings but gave consistent results among tests when isolates were tested multiple times. Determination of incubation period and latent period also required multiple observations. Incubation period was of little value, since significant differences were rarely detected among isolates. This has previously been shown in other studies $(14,26)$. Latent period, on the other hand, was useful in detecting differences among isolates and is probably a better measure of aggressiveness than incubation period because it affects the rate of additional cycles of infection. Differences among isolates were detected with sporulation capacity and lesion size but not as readily as with AULEC.

Aggressiveness tests were begun in the fall of 1994, and there was an initial concern that isolates collected in 1992 and 1993 might have lost aggressiveness during storage. Isolates were there- fore inoculated on tuber tissue and leaflets (cultivar Russet Norkotah) and then reisolated. Inoculum for all aggressiveness tests was increased on tuber tissue to help maintain isolate aggressiveness. This apparently was effective, or not needed, because isolate values for the different components of aggressiveness were consistent in various trials and also because some isolates collected in 1992 (isolate 416) and 1993 (isolate 336) were relatively aggressive.

Screening isolates for components of aggressiveness on detached leaflets was effective and gave consistent results, indicating that differences among treatments resulted from differences in isolates and not in test conditions. For example, isolates 302, 469, 537, and 584 all ranked similarly in aggressiveness when they were analyzed in multiple tests through time. The isolate used to standardize all aggressiveness scores, Pi 94, also ranked consistently low for aggressiveness components. Some differences in aggressiveness scores were caused by interactions between specific isolates and cultivars. The utility of the detached-leaflet assay compared with testing whole plants is that a large number of isolates and more components of aggressiveness can be studied. Additionally, isolates are less likely to become contaminated by other isolates in detached-leaflet assays.

Some traits, such as isolate infectivity, were not tested by the detached-leaflet tests. Additionally, only one infection cycle was observed in these tests. When all of the components of isolate aggressiveness are combined and then multiplied over many infection cycles, larger differences among isolates may appear. Field and greenhouse studies incorporate more components of isolate aggressiveness than detached-leaflet studies, and multiple infection cycles can be observed in field studies.

Selected isolates included in both detached-leaflet and wholeplant studies ranked similarly for relative aggressiveness when compared by Spearman's rank correlation coefficient. The most aggressive US-8 isolate, 584, and the most aggressive US-11 isolate, 537, had similar AUDPC values on whole plants. Additionally, US-1 and US-7 isolates did not differ significantly. Significant differences observed among some isolates for AUDPC on whole plants in the greenhouse were not observed for the same isolates for AULEC, latent period, and sporulation capacity in detached-leaflet assays. Components of disease aggressiveness for

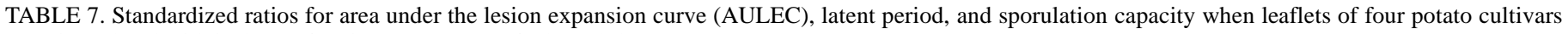
were inoculated with isolates of various genotypes of Phytophthora infestans ${ }^{\mathrm{y}}$

\begin{tabular}{|c|c|c|c|c|c|c|c|c|}
\hline \multirow{2}{*}{$\begin{array}{l}\text { Component } \\
\text { Genotype }\end{array}$} & \multicolumn{2}{|c|}{ Russet Burbank } & \multicolumn{2}{|c|}{ Russet Norkotah } & \multicolumn{2}{|c|}{ Shepody } & \multicolumn{2}{|c|}{ Legend } \\
\hline & Test 1 & Test 2 & Test 1 & Test 2 & Test 1 & Test 2 & Test 1 & Test 2 \\
\hline \multicolumn{9}{|l|}{ AULEC } \\
\hline US-8(1-3) & $2.60 \mathrm{a}$ & $1.93 \mathrm{a}$ & $2.33 \mathrm{a}$ & $2.23 \mathrm{a}$ & $1.71 \mathrm{a}$ & $1.70 \mathrm{a}$ & $1.64 \mathrm{a}$ & $1.67 \mathrm{a}$ \\
\hline US-11(1-2) & $2.04 \mathrm{~b}$ & $2.04 \mathrm{a}$ & $1.53 \mathrm{bc}$ & $2.54 \mathrm{a}$ & $1.60 \mathrm{a}$ & $1.21 \mathrm{~b}$ & $1.57 \mathrm{ab}$ & $1.49 \mathrm{ab}$ \\
\hline Undetermined ${ }^{\mathrm{z}}$ & $1.41 \mathrm{c}$ & $1.51 \mathrm{~b}$ & $1.86 \mathrm{~b}$ & $1.31 \mathrm{~b}$ & $1.25 \mathrm{~b}$ & $1.67 \mathrm{a}$ & $\ldots$ & $\ldots$ \\
\hline US-7(2-3) & $1.53 \mathrm{c}$ & $1.04 \mathrm{c}$ & $1.39 \mathrm{c}$ & $1.50 \mathrm{~b}$ & $1.13 \mathrm{~b}$ & $1.09 \mathrm{bc}$ & $1.39 \mathrm{ab}$ & $1.49 \mathrm{ab}$ \\
\hline US-1(4-5) & $1.29 \mathrm{c}$ & $0.97 \mathrm{c}$ & $1.29 \mathrm{c}$ & $1.29 \mathrm{~b}$ & $0.88 \mathrm{c}$ & $0.95 \mathrm{~d}$ & $1.22 \mathrm{~b}$ & $1.20 \mathrm{bc}$ \\
\hline US-6(2-3) & $1.26 \mathrm{c}$ & $0.91 \mathrm{c}$ & $0.90 \mathrm{~d}$ & $1.36 \mathrm{~b}$ & $0.84 \mathrm{c}$ & $1.02 \mathrm{~cd}$ & $0.61 \mathrm{c}$ & $0.25 \mathrm{c}$ \\
\hline \multicolumn{9}{|l|}{ Latent period } \\
\hline US-8(1-3) & $0.72 \mathrm{ab}$ & $0.72 \mathrm{a}$ & $0.76 \mathrm{a}$ & $0.70 \mathrm{ab}$ & $0.81 \mathrm{ab}$ & $0.81 \mathrm{~b}$ & $0.72 \mathrm{a}$ & 0.72 \\
\hline US-11(1-2) & $0.70 \mathrm{a}$ & $0.69 \mathrm{a}$ & $0.80 \mathrm{a}$ & $0.63 \mathrm{a}$ & $0.73 \mathrm{a}$ & $0.92 \mathrm{~cd}$ & $0.73 \mathrm{a}$ & 0.77 \\
\hline Undetermined ${ }^{\mathrm{z}}$ & $0.99 \mathrm{~cd}$ & $0.73 \mathrm{a}$ & $0.79 \mathrm{a}$ & $0.77 \mathrm{bc}$ & $0.86 \mathrm{bc}$ & $0.68 \mathrm{a}$ & $\cdots$ & $\ldots$ \\
\hline US-7(2-3) & $0.83 \mathrm{bc}$ & $0.91 \mathrm{~b}$ & $0.82 \mathrm{a}$ & $0.77 \mathrm{bc}$ & $0.90 \mathrm{~cd}$ & $0.93 \mathrm{c}$ & $0.75 \mathrm{a}$ & 0.76 \\
\hline US-6(1-4) & $0.96 \mathrm{c}$ & $1.11 \mathrm{~b}$ & $1.22 \mathrm{~b}$ & $0.94 \mathrm{c}$ & $1.11 \mathrm{~d}$ & $1.08 \mathrm{~cd}$ & $1.26 \mathrm{~b}$ & 1.46 \\
\hline US-1(2-5) & $1.02 \mathrm{~d}$ & $1.02 \mathrm{~b}$ & $0.89 \mathrm{a}$ & $0.88 \mathrm{c}$ & $1.16 \mathrm{~d}$ & $1.27 \mathrm{~d}$ & $0.90 \mathrm{a}$ & 0.92 \\
\hline \multicolumn{9}{|c|}{ Sporulation capacity } \\
\hline US-8(1) & $\ldots$ & $\cdots$ & 3.77 & 2.85 & $\ldots$ & $\cdots$ & $20.25 \mathrm{a}$ & $5.16 \mathrm{a}$ \\
\hline US-11(1) & $2.02 \mathrm{a}$ & $\ldots$ & 1.39 & 1.64 & 1.72 & $\ldots$ & $3.46 \mathrm{c}$ & $2.25 \mathrm{ab}$ \\
\hline Undetermined $^{\mathrm{z}}$ & $1.72 \mathrm{ab}$ & $2.91 \mathrm{a}$ & 1.97 & 1.24 & 1.67 & 1.78 & $\ldots$ & $\ldots$ \\
\hline US-7(2) & $2.30 \mathrm{a}$ & $1.70 \mathrm{ab}$ & 1.77 & 2.25 & 1.79 & 2.43 & $8.59 \mathrm{~b}$ & $4.76 \mathrm{ab}$ \\
\hline US-1(2-5) & $2.09 \mathrm{a}$ & $1.30 \mathrm{bc}$ & 2.13 & 1.88 & 1.70 & 1.38 & $10.61 \mathrm{~b}$ & $3.65 \mathrm{ab}$ \\
\hline US-6(1) & $0.84 \mathrm{~b}$ & $0.34 \mathrm{~d}$ & 0.96 & 1.54 & 0.47 & 0.38 & $\ldots$ & $1.80 \mathrm{~b}$ \\
\hline
\end{tabular}

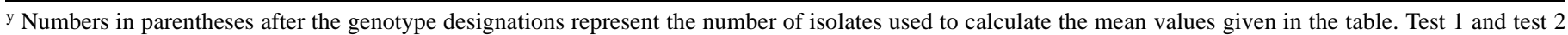
refer to repeats of the same test. Missing data are designated by ellipses. Values followed by the same letter within a column of a subsection do not differ significantly from each other.

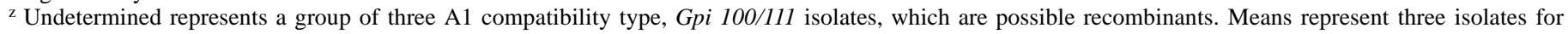
AULEC and latent period and two isolates for sporulation capacity. 
a pathogen population probably act cumulatively in increasing disease, and measurement of one component may not give a total measure of aggressiveness. The detached-leaflet assays detected significant differences among isolates. However, differences were more obvious in the greenhouse tests in which the AUDPC values were used. The greenhouse AUDPC values reflect isolate infectivity through the adaxial leaf surface and subsequent spread through host tissue, whereas infectivity does not play as great a role with detached leaflet AULEC. Results in this study were similar to those of studies dealing with host resistance $(18,19)$. Components of disease resistance are the complement in the host to components of disease aggressiveness in the pathogen (36). Some individual components of partial resistance measured on potted plants in a greenhouse did not differ significantly among resistant and susceptible barley and asparagus cultivars infected with Puccinia hordei and $P$. asparagi, respectively $(18,19)$. Significant differences were detected among the resistant and susceptible cultivars in the field in both studies by using AUDPC, however.

The relative differences in aggressiveness components may partially explain the shift in the $P$. infestans population in the Columbia Basin from the US-1 genotype to the US-8 genotype (28). Goodwin et al. (12) noted that epidemics in North America associated with the appearance of new $P$. infestans genotypes have been more severe than epidemics associated with the US-1 genotype. Almost all isolates collected from the Columbia Basin in 1992 were of the US-1 genotype. By 1995, 97\% of all isolates collected were US-8 isolates (28). During a growing season, more aggressive isolates, such as those of the US-8 strain, would colonize plant tissue faster and sporulate more than isolates of a less aggressive strain. Increased ability to colonize may allow aggressive US-8 isolates to outdo less aggressive strains in the competition for available host tissue. Greater sporulation capacity would also increase the chance of dispersal and dissemination for US-8 isolates. Metalaxyl resistance present in US-8 strains may also be a factor in the shift of the population. The importance of this is unclear, however, since metalaxyl-sensitive isolates were recovered in 1994 after very few metalaxyl-sensitive isolates were found in 1993. Metalaxyl was still being applied one to two times to some fields in the Columbia Basin in 1994 to control storage rot (28). Goodwin et al. (12) point out that the association of recent severe epidemics with new genotypes may be influenced by the occurrence of weather conducive for late blight. This does not exclude the effect of the new genotypes, however.

The isolate by cultivar interactions and higher relative aggressiveness of the newer strains, US-8 and US-11, have important ramifications in developing resistant cultivars and late blight management strategies. Potato genotypes should be exposed to a sufficient number of isolates of $P$. infestans to ensure that newly developed cultivars with partial resistance are effective against a wide spectrum of pathogen biotypes. The present guidelines for managing potato late blight were developed while the old US-1 strain existed exclusively. Because of the increased aggressiveness of newer strains, which are now widespread $(9,10,12$, 28,33 ), current management practices need to be modified. Since tissue colonization by US-8 and US-11 genotypes is greater and since US-8 isolates sporulate more abundantly than other strains, more intense management practices will need to be adopted in potato-producing areas where these genotypes are present. Kato et al. (23) showed by simulations analysis that using a 7-day spray interval to control a late blight epidemic caused by the US- 8 genotype was equivalent to using a 9- to 10-day spray interval to control a late blight epidemic caused by the US-1 genotype. Fungicide spray intervals may need to be shortened when dealing with epidemics caused by the US-8 genotype, and initial fungicide applications may need to be earlier than in the past.

\section{ACKNOWLEDGMENTS}

Plant Pathology New Series 0250, Project 0678, Department of Plant Pathology, College of Agriculture and Home Economics Research Center, Pullman, WA. We thank J. R. Alldredge, Program in Statistics, Washington State University, for statistical consultations; B. A. Fry for help in genotype identification of isolates; G. Pelter, T. Cummings, K. Pritchard, and J. Jaeger for technical assistance; and R. C. Rowe, D. A. Inglis, and L. M. Carris for their valuable comments and suggestions for the manuscript.

\section{LITERATURE CITED}

1. Anonymous. 1947. Discover blight menace to late potato crops. Prosser Record-Bulletin, Prosser, WA. 25 September. p. 1.

2. Bashan, B., Kadish, D., Levy, Y., and Cohen, Y. 1989. Infectivity to potato, sporangial germination, and respiration of isolates of Phytophthora infestans from metalaxyl-sensitive and metalaxyl-resistant populations. Phytopathology 79:832-836.

3. Castronovo, A. H., Thurston, D., and Eide, C. J. 1954. Parasitic aggressiveness and its relation to late blight tolerant potato varieties and the survival of the pathogen. (Abstr.) Am. Potato J. 31:366-367.

4. Cohen, Y. 1994. Local and systemic control of Phytophthora infestans in tomato plants by DL-3-amino-n-butanoic acids. Phytopathology 84:5559.

5. Cohen, Y., Farkash, S., Reshit, Z., and Baider, A. 1997. Oospore production of Phytophthora infestans in potato and tomato leaves. Phytopathology 87:191-196.

6. Day, J. P., and Shattock, R. C. 1997. Aggressiveness and other factors relating to displacement of populations of Phytophthora infestans in England and Wales. Eur. J. Plant Pathol. 103:379-391.

7. Deahl, K. L., DeMuth, S. P., Pelter, G., and Ormrod, D. J. 1993. First report of resistance of Phytophthora infestans to metalaxyl in eastern Washington and southwestern British Columbia. Plant Dis. 77:429.

8. Deahl, K. L., Inglis, D. A., and DeMuth, S. P. 1993. Testing for resistance to metalaxyl in Phytophthora infestans isolates from northwestern Washington. Am. Potato J. 70:779-795.

9. Fry, W. E., Goodwin, S. B., Dyer, A. T., Matuszak, J. M., Drenth, A., Tooley, P. W., Sujkowski, L. S., Koh, Y. J., Cohen, B. A., Spielman, L. J., Deahl, K. L., Inglis, D. A., and Sandlan, K. P. 1993. Historical and recent migrations of Phytophthora infestans: Chronology, pathways, and implications. Plant Dis. 77:653-661.

10. Goodwin, S. B., Cohen, B. A., Deahl, K. L., and Fry, W. E. 1994. Migration from northern Mexico as the probable cause of recent genetic changes in populations of Phytophthora infestans in the United States and Canada. Phytopathology 84:553-558.

11. Goodwin, S. B., Schneider, R. E., and Fry, W. E. 1995. Use of celluloseacetate electrophoresis for rapid identification of allozyme genotypes of Phytophthora infestans. Plant Dis. 79:1181-1185.

12. Goodwin, S. B., Sujkowski, L. S., Dyer, A. T., Fry, B. A., and Fry, W. E 1995. Direct detection of gene flow and probable sexual reproduction of Phytophthora infestans in northern North America. Phytopathology 85: 473-479.

13. Goodwin, S. B., Sujkowski, L. S., and Fry, W. E. 1995. Rapid evolution of pathogenicity within clonal lineages of the potato late blight disease fungus. Phytopathology 85:669-676.

14. Guzmán-N., J. 1964. Nature of partial resistance of certain clones of three Solanum species to Phytophthora infestans. Phytopathology 54:13981404.

15. Hamm, P. B., Fry, B. A., and Jaeger, J. 1994. Occurrence and frequency of metalaxyl insensitivity and mating types of Phytophthora infestans in the Columbia Basin of Oregon and Washington. (Abstr.) Phytopathology 84:1123.

16. Hodgson, W. A. 1961. Laboratory testing of the potato for partial resistance to Phytophthora infestans. Am. Potato J. 38:259-264.

17. Inglis, D. A., Johnson, D. A., Legard, D. E., Fry, W. E., and Hamm, P. B. 1996. Relative resistances of potato clones in response to new and old populations of Phytophthora infestans. Plant Dis. 80:575-578.

18. Johnson, D. A. 1986. Two components of slow-rusting in asparagus infected with Puccinia asparagi. Phytopathology 76:208-211.

19. Johnson, D. A., and Wilcoxson, R. D. 1978. Components of slow-rusting in barley infected with Puccinia hordei. Phytopathology 68:1470-1474.

20. Kadish, D., and Cohen, Y. 1988. Fitness of Phytophthora infestans isolates from metalaxyl-sensitive and -resistant populations. Phytopathology 78:912-915.

21. Kadish, D., and Cohen, Y. 1989. Population dynamics of metalaxylsensitive and metalaxyl-resistant isolates of Phytophthora infestans in untreated crops of potato. Plant Pathol. 38:271-276.

22. Kadish, D., Grinberger, M., and Cohen, Y. 1990. Fitness of metalaxylsensitive and metalaxyl-resistant isolates of Phytophthora infestans on 
susceptible and resistant potato cultivars. Phytopathology 80:200-205.

23. Kato, M., Mizubuti, E. S., Goodwin, S. B., and Fry, W. E. 1997. Sensitivity to protectant fungicides and pathogenic fitness of clonal lineages of Phytophthora infestans in the United States. Phytopathology 87:973-978.

24. Knutson, K. W., and Eide, C. J. 1961. Parasitic aggressiveness in Phytophthora infestans. Phytopathology 51:286-290.

25. Lambert, D. H., and Currier, A. I. 1997. Differences in tuber rot development for North American clones of Phytophthora infestans. Am. Potato J. 74:39-43.

26. Lapwood, D. H. 1961. Potato haulm resistance. II. Lesion production and sporulation. Ann. Appl. Biol. 49:316-330.

27. Lapwood, D. H., and McKee, R. K. 1966. Dose-response relationships for infection of potato leaves by zoospores of Phytophthora infestans. Trans. Br. Mycol. Soc. 49:679-686.

28. Miller, J. S., Hamm, P. B., and Johnson, D. A. 1997. Characterization of the Phytophthora infestans population in the Columbia Basin of Oregon and Washington from 1992 to 1995. Phytopathology 87:656-660.

29. Ribeiro, O. K. 1978. A Source Book of the Genus Phytophthora. J. Cramer, Vaduz, Germany.

30. Rivera-Peňa, A. 1990. Wild tuber-bearing species of Solanum and inci- dence of Phytophthora infestans (Mont.) de Bary on the western slopes of the volcano Nevado de Toluca. 5. Type of resistance to $P$. infestans. Potato Res. 33:479-486.

31. Shaner, G. 1980. Probits for analyzing latent period data in studies of slow rusting resistance. Phytopathology 70:1179-1182.

32. Stewart, H. E. 1990. Effect of plant age and inoculum concentration on expression of major gene resistance to Phytophthora infestans in detached potato leaflets. Mycol. Res. 94:823-826.

33. Sujkowski, L. S., Goodwin, S. B., Dyer, A. T., and Fry, W. E. 1994. Increased genotypic diversity via migration and possible occurrence of sexual reproduction of Phytophthora infestans in Poland. Phytopathology 84:201-207.

34. Tooley, P. W. 1990. Variation in resistance to Phytophthora infestans among 21 Solanum verrucosum plant introductions. Am. Potato J. 67: 491-498.

35. Tooley, P. W., Sweigard, J. A., and Fry, W. E. 1986. Fitness and virulence of Phytophthora infestans isolates from sexual and asexual populations. Phytopathology 76:1209-1212.

36. Vanderplank, J. E. 1968. Disease Resistance in Plants. Academic Press, New York. 